\title{
Der Apfel fällt nicht weit vom Stamm? Die werbliche Darstellung von Jugend- und Elterngeneration im Vergleich
}

\author{
Julia Maria Derra, Cornelia Eck und Michael Jäckel
}

\begin{abstract}
Geschlechterinszenierungen in Werbeanzeigen sind spätestens seit Goffman (engl. Original 1976 / dt. Übersetzung 1981) ein beliebtes Forschungsthema. Dabei wurde ein expliziter Generationenvergleich bisher jedoch vernachlässigt. Eine inhaltsanalytische Untersuchung der werblichen Inszenierung von Männern und Frauen mit dem Schwerpunkt auf einem Vergleich der Jugend- und Elterngeneration (hier: 14- bis 19-Jährige vs. 40- bis 49-Jährige) zeigt, dass die Anzeigenwerbung heute einerseits auf veränderte gesellschaftliche Rahmenbedingungen reagiert und mit traditionellen Darstellungsprinzipien gebrochen hat. Andererseits wird ersichtlich, dass gerade jugendliche Werbeprotagonisten/-innen in ein Werbekorsett der Äusserlichkeiten eingepasst und stark genormt werden. Die Generation 40-49 besitzt dagegen bezüglich ihres Äusseren mehr Freiräume, ist jedoch auf anderen Ebenen, z. B. im Bereich der beworbenen Produkte, weiterhin an traditionell geschlechtlich bestimmte Rahmungen gebunden.
\end{abstract}

\section{Medien als Mitgestalter der Gesellschaft}

Die Zahl medialer Kommunikationsformen wächst unaufhörlich; Medien differenzieren sich immer weiter aus. Damit steigt auch die Quantität verfügbarer Informationen und Materialien, die individuell rezipiert und genutzt werden können. Die Entgrenzung von räumlichen und zeitlichen Bedingungen medial vermittelter Kommunikation erzeugt eine Allgegenwart und eine wachsende Vermischung von Medienwirklichkeit und sozialer Wirklichkeit. Veränderte gesellschaftliche Kommunikation und kommunikativ konstruierte Wirklichkeit sind die Folge. Friedrich Krotz (2007; vgl. auch Krotz 2008: 52; Thomas \& Krotz 2008: 28f) spricht in diesem Zusammenhang von dem Prozess der «Mediatisierung». Soziales Handeln geschieht heute somit in einer medial-geprägten Umwelt und lässt sich ohne Bezug auf den Mediatisierungsprozess nicht fassen (vgl. Thomas \& Krotz 2008: 29; Krotz 2008: $55 f$ ).

Medien müssen als «Mitgestalter` der Gesellschaft angesehen werden, die in die Wirklichkeit integriert sind und mit ihr interagieren. Besonders die Vermischung medialer und sozialer Kommunikation gilt heute als wichtige Komponente der Identitätsentwicklung und Identitätssicherung, zur Bestärkung persönlicher Werthaltungen sowie zur Suche nach Verhaltensmodellen (vgl. z. B. Mikos 2005; Schorb 2006). Normen, Werte und Rollenbilder werden nicht mehr nur anhand «leibhaf- 
tigers Vorbilder in der direkten sozialen Umgebung gelernt, sondern gerade die Identifikation mit oder Abgrenzung von Personen und Szenen aus Medienepisoden ermöglichen es Rezipienten, sich selbst neu kennen und verstehen zu lernen. Medien haben sich längst als Sozialisationsinstanz etabliert. Sie werden heute als untrennbar mit dem Identitätskonzept verknüpft angesehen, da sie ein breitgefächertes Angebot an Identitätsmustern liefern (vgl. u. a. Krotz 2003: 42; Mikos 2005; Schorb 2006). Eine Grenzziehung zwischen realer und medialer Welt wird dabei immer schwieriger und erfordert immer höhere Kompetenzen (vgl. Körber \& Schaffar 2003: 82).

Auch im medienübergreifenden Genre «Werbung» sind es "zweifellos nicht immer nur die Produkte selbst, sondern vielmehr die mit den beworbenen Produkten verknüpften Lebenskontexte und Lebensstile» (Ferchhoff 1999: 237), die auf Interesse stossen. Werbung tritt als Sozialisationsagent auf, vermittelt Wertvorstellungen und Verhaltensmuster, gibt Orientierungs- und Lebenshilfen und kann als Instrument der Wirklichkeitskonstruktion sowohl für das individuelle als auch für das kollektive Gedächtnis angesehen werden (vgl. Spiess 1992: 91; Luca 2003).

Besonders die Entwicklungsphase Jugend - gekennzeichnet durch eine zunehmende Ablösung von den Eltern einhergehend mit dem erhöhten Einfluss ausserhäuslicher Sozialisationsinstanzen - ist geprägt durch eine zentrale Auseinandersetzung mit der Identität, dem eigenen Aussehen sowie der Suche nach Vorbildern. Der Einfluss von Peers und Medienfiguren nimmt im Jugendalter zu, dennoch spielen Eltern im Leben von Jugendlichen weiterhin als Ratgeber eine wichtige Rolle (vgl. Oerter \& Dreher 1998: 362, 384; Mikos 2005: 21-23; Geulen 2007: 152). Jugendliche und ihre Eltern, zwei Generationen, die also einerseits stark miteinander verbunden sind, sich andererseits hinsichtlich ihrer Lebensphasen, des Standes ihrer Persönlichkeitsentwicklung und ihrer Bedürfnisse elementar voneinander unterscheiden. Der Generationenbegriff wird im Folgenden dennoch stärker im bevölkerungsstatistischen Sinne verstanden, also als durchschnittlicher Abstand zwischen den Geburtsjahren der Eltern und ihrer Kinder, und nicht nach soziologischer Definition als gleichaltrige Personen mit ähnlichen kulturellen und sozialen Einstellungen sowie Verhaltensweisen (vgl. Hillmann 2007: 274).

Ein Inszenierungsvergleich der Jugend- und Elterngeneration verknüpft mit der Frage, inwiefern die Werbung auf generationsspezifische Eigenheiten reagiert oder ob die Redewendung ‘Der Apfel fällt nicht weit vom Stamm〉 auf die werbliche Inszenierung dieser beiden miteinander verbundenen Generationen übertragen werden kann, verspricht neue Erkenntnisse in der Werbeforschung. Auch mit Blick auf die veränderte Altersverteilung der Gesellschaft, die mit einem neuen Selbstverständnis verschiedener Generationen sowie einer zunehmenden Zielgruppendifferenzierung in Werbewirtschaft und Marketing einhergeht, erscheint eine solche Analyse immer relevanter. Bisher werden geschlechtsspezifische Darstellungsprinzipien - von wenigen Ausnahmen (vgl. z. B. Hastenteufel 1980) abge- 
sehen - jedoch ohne Berücksichtigung der Generationenzugehörigkeit betrachtet. Deshalb stehen die werblichen Darstellungsstrategien von Jugendlichen und Eltern im Zentrum einer aktuellen inhaltsanalytischen Untersuchung. ${ }^{1}$

Als Jugendliche wurden dabei alle Protagonisten/innen im Alter von 14-19 Jahren bezeichnet. Die Zusammenfassung verschiedener Entwicklungsstufen in der Jugendphase war notwendig, um eine ausreichend grosse Fallzahl für die inhaltsanalytische Untersuchung zu erzielen und die Schwierigkeit der genauen Alterseinschätzung, die bei Heranwachsenden besonders gross ist, weitestgehend zu vermeiden. Da die Mütter der heute 14- bis 19-Jährigen laut Statistischem Bundesamt bei der Geburt durchschnittlich 28,5 Jahre alt waren (vgl. Statistisches Bundesamt 2007: 30f), wurde als zugehörige Elterngeneration die Altersklasse der 40- bis 49-Jährigen definiert.

Da zentrale Aussagen und Kritikpunkte vorhergehender Studien zu werblichen Geschlechterinszenierungen durchaus für die eigene Analyse Relevanz besitzen, soll zunächst ein kurzer Forschungsüberblick zum Thema (Werbung und Geschlecht) gegeben werden.

\section{Werbung und Geschlecht}

Etwa seit den 60er Jahren des letzten Jahrhunderts gibt es Analysen zu Geschlechterinszenierungen in der Werbung. Die Untersuchung werblicher Geschlechterkonstruktionen ist für die Sozialwissenschaften deshalb so interessant, weil sich daran z. B. wandelnde Schönheitsideale oder Rollenbilder ablesen lassen (vgl. u. a. Schmidt 2000). Die anfänglichen Untersuchungen konzentrieren sich jedoch meist auf die Darstellung der Frau und sind geprägt durch einen emanzipatorischen Blickwinkel (vgl. z. B. Schmerl u.a. 1984). Erst deutlich später, etwa seit den 1990er Jahren, rückt auch die werbliche Inszenierung der Männer in den wissenschaftlichen Fokus (vgl. z. B. Krohne 1995; Zurstiege 1998).

Erving Goffman kommt Ende der 1970er Jahre in seiner Analyse geschlechtsspezifischer Darstellungen in Reklameanzeigen zu der Feststellung, dass werbliche Geschlechterkonstruktionen formalisierte und stereotypisierte Verhaltensweisen liefern, die wie Signale wirken und damit eine leichtere Lesbarkeit der Situation ermöglichen (Goffman 1981: 9, 31).

Um die Brücke zwischen der Darstellung der Geschlechter in der Gesellschaft und in der Reklame zu schlagen, soll an dieser Stelle kurz der theoretische Hintergrund skizziert werden: Goffman geht davon aus, dass sich der Mensch in sozialen Situationen wechselseitig durch die Gesamtheit seines Verhaltens und seiner Erscheinung über seine soziale Identität, Stimmung, Absicht, Erwartungen und den Stand der Beziehung informiert. Auf diese Weise wird der Stil des Verkehrs miteinander

Grundlage dieser empirischen Analyse sind Daten, die im Rahmen des Drittmittelprojekts «Männlich und Weiblich im Spiegel der Werbung» erfasst wurden. Das Projekt wurde vom 1. Juli 2006 bis zum 31. Oktober 2008 an der Universität Trier (Fachbereich IV, Soziologie) durchgeführt. 
festgelegt (vgl. Goffman 1981: 9f). Die Verhaltensweisen, die der Mensch dabei verwendet, sind nicht beliebig, sondern bestimmte, kulturell verschiedene, formalisierte Elemente mit dem Zweck, die Situation für den Interaktionspartner/die Interaktionspartnerin leichter lesbar zu machen. Diese stereotypisierten bzw. ritualisierten Verhaltensweisen nennt Goffman in Anlehnung an Julian Huxley «Darstellungen> (vgl. Goffman 1981: 8f).

- Weiblichkeit und Männlichkeit sind Prototypen des essentiellen Ausdrucks, d. h., sie gehören zu den am tiefsten verankerten Merkmalen eines Menschen (vgl. Goffman 1981: 34). Goffman geht jedoch davon aus, dass die Darstellungen der einzelnen Geschlechter keinen biologischen Ursprung haben, sondern dass ihre Wurzeln in der Kultur zu suchen sind (vgl. Goffman 1981: 19). Männer und Frauen verfahren bei der Darbietung ihres (sozialen) Geschlechts nach einem kulturell festgelegten Plan (vgl. Goffman 1981: 37; Willems \& Jurga 1998: 181f). Dieser orientiert sich u. a. an Unterwerfungsgesten (vgl. Goffman 1981: 20, 165) und am Eltern-Kind-Komplex, auf den im Folgenden näher eingegangen wird (vgl. zu den folgenden Ausführungen: Goffman 1981: 20-28; Goffman bezieht sich dabei auf die ideale Eltern-Kind-Beziehung in der Mittelschicht):

- Das Besondere am Eltern-Kind-Komplex ist, dass die meisten Menschen im Verlauf ihres Lebens beide Rollen erfahren und es sich damit um ein geschlechtsneutrales Darstellungsmittel handelt. Die Rolle des Kindes ist mit verschiedenen Vor- und Nachteilen verbunden. So müssen sich Kinder dank ihrer Eltern nicht auf die Notwendigkeiten und Zwänge der sozialen Situationen einlassen, sondern brauchen dies nur spielerisch zu tun; «für sie ist die Realität eine verzeihende.» (Goffman 1981: 24). Aber dafür unterliegen sie auch der Kontrolle der Älteren, müssen Entscheidungen über ihren Kopf hinweg und die freie Verfügung über ihre Zeit akzeptieren. Diese Erfahrungen bzw. Verhaltensweisen übertragen wir auf unsere Begegnungen mit Erwachsenen und offenbar übernimmt der Mann dabei immer die Eltern-, d. h. Beschützerrolle, und die Frau die Kinderrolle².

Die Werbung greift nun solche ohnehin schon ritualisierten Verhaltensweisen auf und stilisiert sie erneut. Dieses Prinzip bezeichnet Goffman mit dem Begriff «Hyper-Ritualisierung> (vgl. Goffman 1981: 328; Willems \& Jurga 1998: 182f). Das Ziel, das die Werbedesigner damit erreichen möchten, ist das gleiche, das auch der Mensch in sozialen Situationen anstrebt, nämlich die eigenen Handlungen und sich selbst für andere verständlich zu machen. «Und doch - ob wir nun für ein Bild posieren oder wirklich eine rituelle Handlung ausführen - stets zeigen wir ein Re-

In Situationen mit gleichgeschlechtlichen Teilnehmern/Teilnehmerinnen «beschützt〉 die statushöhere Person. 
klamebild, eine ideale Darstellung, die beschwört, wie die Dinge eigentlich sein sollten.» (Goffman 1981: 328)

Das System der Werbung funktioniert also auf Basis geschlechtsklassenspezifischer Zeichen, die Aspekte für Weiblichkeit und Männlichkeit involvieren. Männer und Frauen werden dabei jedoch nicht einfach nur dargestellt, sondern es werden Vorstellungen darüber präsentiert, wie sie sind bzw. sein sollen (vgl. Forster 1995: 57; Zurstiege 1998: 198ff). Solch stereotype Vorstellungen gewährleisten ein einfaches und somit schnelles Verständnis der Werbebotschaft, weshalb ihr Einsatz für Werbetreibende besonders lohnend ist. Geht es doch gerade in den Medien darum, Informationen zu vermitteln, die auf den ersten Blick verständlich sind, da die Möglichkeit der Nachfrage in der Regel entfällt. Es ist daher davon auszugehen, dass es für die Werbung besonders zweckmässig ist, das System der Zweigeschlechtlichkeit aufrecht zu erhalten und als Mass für die gesellschaftliche Bedeutung von Geschlechtstypen zu fungieren. Nach Luhmann (2004: 28) ist nämlich gerade die "öffentliche Rekursivität (...), die Voraussetzung des Schon-BekanntSeins und des Bedarfs für weitere Informationen, ein typisches Produkt (...) massenmedialer Kommunikation».

Stereotype Darstellungen werden daher in Werbeanalysen immer wieder bestätigt: Die Frau als fürsorgliche Hausfrau ohne Sex-Appeal oder jung, schön und begehrt, allgemein beschränkt auf passive, dienende, aufreizende oder ästhetische Funktionen (vgl. z. B. Schmerl 1984: 100ff; Bergler u. a. 1992: 31ff), der Mann dagegen als respektiertes Familienoberhaupt oder kompetenter Fachmann, erfolgreich, sachlich und aktiv (vgl. u. a. Krohne 1995: 140f).

Gleichzeitig ist spätestens seit den 1990er Jahren auch eine Ausdifferenzierung und Loslösung von stereotypen Darstellungsprinzipien erkennbar (vgl. Bergler u. a. 1992; Spiess 1992, 1994; Mühlen Achs 1995; Krohne 1995; Zurstiege 1998; Willems \& Jurga 1998; Willems \& Kautt 1999, 2000). Spiess spricht im Bezug auf das weibliche Geschlecht z. B. von dem Typ der selbstbewussten, sich selbstverwirklichenden Frau, welche die Rolle der aktiven Verführerin spielt, ohne sich dabei jedoch verführen zu lassen (vgl. Spiess 1992: 101; oder auch die «neue Müssiggängerin», Schmerl 1994: 135).

Aber auch neue werbliche Rollenentwürfe bleiben selten frei von Kritik. Die Einwände richten sich einerseits gegen die Gefahr, dass vor allem pubertierenden Jungen und Mädchen von den Medien keine qualitativ differenzierten Frauen- und Männerbilder präsentiert werden als die als vorherrschend beschriebenen und besonders Mädchen im Zuge der Selbstsuche somit keine bemerkenswerten Anregungen und Hilfestellungen bekommen, um ein anderes, positives Frauenbild für sich herauszufiltern (vgl. Bergler u. a. 1992: 37; Schmerl 1994: 145). Andererseits wird von den Forschern/innen das Vorführen von perfekten Charakteren, Körpern und Lebensstilen beanstandet, das ein Streben nach unerreichbaren Idealen aus- 
lösen kann (vgl. Schmerl 1984; Baacke u. a. 1993: 210-227; Bieber-Delfosse 2002: 112f; Luca 2003).

Obwohl Schönheit in erster Linie immer noch als «Frauenschönheit) formuliert wird, werden auch Männerdarstellungen zunehmend dem Schönheitsdiktat unterworfen (entblösster Mann als "Objekt der Begierde», Krohne 1995: 148). In der wachsenden Zahl an Magazinen zum Thema Fitness (z. B. Men's Health, Fit for Fun) wird Männlichkeit bzw. männliche Schönheit gleichgesetzt mit dem Typ des Modellathleten, gekennzeichnet durch die Überbetonung von Muskeln und einen enthaarten, durchtrainierten Oberkörper (vgl. Analyse der Zeitschrift Men's Health bei Kleinschmidt 2003). Auch bei Männern sind also Körper und Aussehen als Selbstdarstellungsinstrumente ins Zentrum der Werbung gerückt, mit der Folge einer entsprechenden Aufwertung durch die Zielgruppe. Die Zunahme krankhafter Phänomene wie Anorexien, übersteigerte Diäten und Operationen, die vorher vorwiegend beim weiblichen Geschlecht auftraten, aber auch der Missbrauch von Anabolika und Steroiden in Verbindung mit übermässigem Fitness-Training können Folgen medial verherrlichter Körperperfektion und der Nichterfüllung des Ideals sein (vgl. Bundeszentrale für gesundheitliche Aufklärung 2008).

Problematisch ist nicht die Schönheitskonstruktion an sich. Problematisch wird dieses Konstrukt, wenn «das eigene Selbstkonzept als Bezugssystem zur Verarbeitung von Werbung» (Bergler u. a. 1992: 35) herangezogen wird und die erlebte Divergenz zwischen dem Fremdbild und dem eigenen Selbstbild zu einer Veränderung des Selbstwert- und Körpergefühls führt (vgl. Bergler u. a. 1992: 171ff). Medien- und Werbefiguren können die Bedeutung von Wunschbildern erhalten und so zu (problematischen) Vorbildern bei individuellen Orientierungsaufgaben werden (vgl. Luca 2003).

\section{Jugendliche und Eltern im Spiegel der Werbung}

\section{Datengrundlage der Untersuchung}

Für die Inhaltsanalyse ${ }^{3}$ wurden bewusst Werbeanzeigen aus dem Printmedium ¿Zeitschrift〉 als Untersuchungsgrundlage ausgewählt. Zeitschriften erfreuen sich grosser Beliebtheit; fast alle Deutschen nutzen sie (vgl. Media Analyse Pressemedien I 2006). Bei Werbeanzeigen handelt es sich zudem nicht um als störend empfundene «Unterbrecherwerbung» (Baacke u. a 1999: 110), sondern es bleibt den Rezipienten/innen überlassen, sich mit der Anzeigenseite eingehender zu beschäftigen oder sie zu 〈überblättern〉, aber sie kann nicht, wie beispielsweise das Fernsehprogramm, umgeschaltet und damit vollkommen ignoriert werden. Da die

\footnotetext{
Nach Früh definiert sich eine Inhaltsanalyse «als eine empirische Methode zur systematischen und intersubjektiv nachvollziehbaren Beschreibung inhaltlicher und formaler Merkmale von Mitteilungen (meist zum Zwecke einer darauf aufbauenden, interpretativen und/oder durch Zusatzkriterien gestützten Inferenz)» (kursiv i. Org., Früh 1998: 107).
} 
Mischung der Anzeigen mit dem redaktionellen Teil dazu verleitet, sie zu betrachten, ist die Rezeption von Werbeanzeigen wahrscheinlicher als die von Werbesendungen im Fernsehen (vgl. Sowinski 1998: 80ff; Baacke u. a. 1999: 109f).

Bei der Auswahl der Zeitschriften dienten die Ergebnisse der IVW 1/2006 und der Media Analyse Pressemedien I 2006 bzgl. Reichweite gesamt und Reichweite Zielgruppe als Entscheidungsgrundlage. Mit ihrer Hilfe wurden vier Frauenzeitschriften, vier Männerzeitschriften und drei sogenannte General-Interest-Zeitschriften mit heterogen-geschlechtlichem Publikum ermittelt. Die zielgruppenspezifische Auswahl der Publikumszeitschriften orientiert sich an den ausgewählten Generationen, die im Fokus der Untersuchung liegen. So wurden für jede der drei Gattungen Zeitschriften mit einer Hauptleserschaft im Alter von 14-19 Jahren und 40-49 Jahren selektiert, um auch diesbezüglich ein möglichst repräsentatives Geschlechterbild zu erhalten. Die Entscheidung fiel auf Bild der Frau, Brigitte, Joy und Tina als Frauenzeitschriften, Auto Bild, Computer Bild, Computer Bild Spiele und Sport Bild als Männerzeitschriften sowie Bravo, Focus und Stern als Zeitschriften mit heterogen-geschlechtlichem Publikum. Neun Ausgaben je Zeitschriftentitel, gleichmässig verteilt auf die Jahre 2004 bis 2006, gelangten in die Stichprobe, die somit insgesamt 99 Zeitschriften umfasst.

Der Codierbogen wurde in Anlehnung an Erving Goffmans «Geschlecht und Werbung» (1981) sowie andere empirische Studien von Hastenteufel (1980), Zurstiege (1998) oder Wilk (2002) entworfen und auf Grundlage eigener Forschungsarbeiten (vgl. Derra 2006; Eck 2008; Derra \& Jäckel 2009; Eck \& Jäckel 2009) weiterentwickelt. Er lässt sich in zwei thematische Einheiten unterteilen: Der erste Teil enthält anzeigenbezogene Variablen, mit denen z. B. die beworbene Produktart oder das szenische Umfeld erfasst wurden. Der zweite Teil beinhaltet personenbezogene Variablen, mit denen etwa das Aussehen, die Kleidung oder die Tätigkeit der codierten Person festgehalten wurden. Insgesamt umfasst der Codierbogen 40 Variablen mit einer unterschiedlichen Anzahl von Ausprägungen. Eine vollständige Darstellung des Kategoriensystems ist deshalb im Rahmen dieses Artikels nicht möglich. ${ }^{4}$

Um eine Verzerrung durch einseitige geschlechtliche Bewertungseinflüsse zu vermeiden und den Grad der Validität der Untersuchungsergebnisse zu erhöhen, wurde ein heterogen-geschlechtliches Codierteam mit zwei Männern und zwei Frauen gebildet. Durch Codiererschulungen und umfangreiche Pretests konnte die Intercoderreliabilität bis zu einer durchschnittlichen Rate von 82 \% gesteigert werden. Die Codierung erfolgte von Dezember 2006 bis Januar 2007. In dieser Zeit wurden alle mindestens halbseitigen Werbeanzeigen mit ausreichend grossen Personenabbildungen ab 14 Jahren inhaltsanalytisch erfasst.

\footnotetext{
4 Um den Ansprüchen der Objektivität bzw. der Nachvollziehbarkeit dennoch zu genügen, ist der
} Codierbogen unter folgender URL einsehbar: http://www.uni-trier.de/index.php?id=6191. 


\section{Forschungsfragen}

Bei der Analyse der Unterschiede bzw. Gemeinsamkeiten in der werblichen Inszenierung der beiden Generationen standen zwei Forschungsfragen im Vordergrund: Da bisherige Studien immer wieder darauf verweisen, dass geschlechtsstereotype Darstellungen in der Werbung weiterhin Bestand haben, wurde in der vorliegenden Analyse untersucht, ob traditionelle Geschlechterstereotype in den beiden ausgewählten Generationen gleichermassen geschlechtsspezifisch verankert sind. Aufgrund der andauernden Kritik gegen die stets schlanken, schönen und jungen Werbefiguren wurde ausserdem analysiert, wie stark die Vertreter/innen der beiden Generationen von der Werbung idealisiert werden.

Die Ergebnisse zu diesen Forschungsfragen werden in den nächsten beiden Abschnitten anhand exemplarischer Beispiele erläutert. Dabei geht es nicht darum, Alterseffekte festzustellen, sondern die beiden ausgewählten Generationen miteinander zu vergleichen. Wenn Unterschiede in der Darstellung der beiden Generationen aufgezeigt werden, handelt es sich nicht um kontinuierliche Effekte, die über alle Altersklassen hinweg, also im Sinne eines Alterseffekts feststellbar sind. Nur eine Längsschnittuntersuchung könnte letztlich Aufschluss darüber geben, inwiefern Unterschiede möglicherweise perioden- (Jahreseffekte) oder kohorteneffektbedingt (Jahrgangseffekte) auftreten.

\section{Die Verteilung Jugendlicher und Eltern auf Werbeanzeigen}

Die Studie beruft sich auf eine Gesamtzahl von 1406 untersuchten Werbeanzeigen; auf 195 dieser Anzeigen sind Jugendliche abgebildet (rund 14\%), auf 169 Anzeigen Eltern (12\%).

Da zur Klärung der Forschungsfragen die Betrachtung einzelner Personen erforderlich war, wurde der Datensatz transformiert und jede Person als Fall definiert. Somit wurden insgesamt 1861 Personen als Fälle erfasst, rund 62 \% davon Frauen, 38 \% Männer. Wie in Abb. 1 erkennbar, sind die beiden im Fokus der Untersuchung stehenden Altersgruppen, die 14- bis 19-Jährigen und die 40- bis 49-Jährigen, mit 13 zu 10 \% (247 14- bis 19-Jährige zu 189 40- bis 49-Jährigen) etwa gleich stark auf den untersuchten Werbeanzeigen vertreten. ${ }^{5}$

\footnotetext{
5 Im Folgenden werden ausschliesslich die Verteilungen der erhobenen Merkmale in den untersuchten Generationen betrachtet. Auf die empirische Verteilung in der Gesamtstichprobe wird in diesem Rahmen nicht weiter eingegangen, da dies für die Forschungsfragen von nachrangiger Bedeutung ist.
} 


\section{Generationenverteilung}

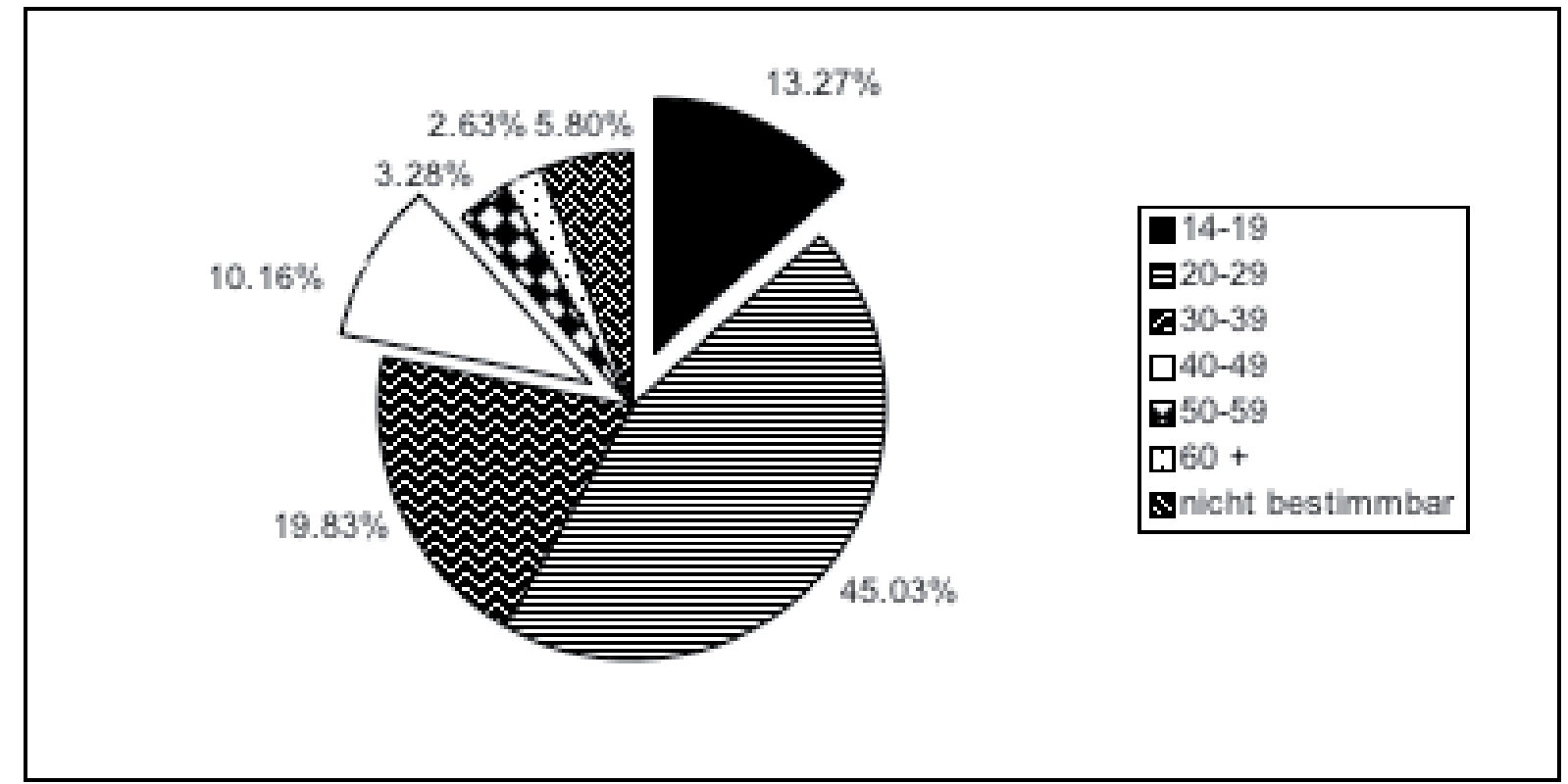

Abb. 1: Altersgruppenverteilung

Signifikant anders $(p=.00 \text {, Phi }=.41)^{6}$ ist dagegen die Geschlechterverteilung: Bei den 14- bis 19-Jährigen werden Frauen (insgesamt 188) mit 76 zu $24 \%$ wesentlich häufiger auf Werbeanzeigen abgebildet als Männer (insgesamt 59). Bei den 40bis 49-Jährigen ist das Verhältnis genau umgekehrt. Nur 35 \% dieser Altersklasse sind weiblich (insgesamt 67), der Grossteil ist dagegen männlich (insgesamt 122). Dieses Ergebnis weist auf eine bekannte Werbestrategie hin: Auch wenn sich die Darstellungen qualitativ gewandelt haben, sind ältere Frauen in der Werbung nach wie vor unterrepräsentiert (vgl. Hastenteufel 1980; Bergler u. a. 1992: 33; RöhrSendlmeier \& Ueing 2004; einen Überblick gibt Jäckel 2009), denn «Frau-Sein (...) bedeutet gleichzeitig Jugendlichkeit» (Eck 2008: 104f) - und die endet werblich betrachtet für Frauen bereits mit dem 40. Lebensjahr. Männer hingegen dürfen altern - «zumindest bis zum 50. Lebensjahr» (Eck 2008: 104).

6 Der Phi-Koeffizient und Cramer's-V sind Zusammenhangsmasse für nominale Daten auf der Basis der Chi-Quadrat-Statistik. Phi ergibt sich als Wurzel aus dem Quotienten aus Chi-Quadrat und dem Stichprobenumfang und wird bei Vierfeldertabellen eingesetzt. Cramer's-V wird bei mehr als zwei nominalskalierten Merkmalen verwendet, weil sein numerischer Wert nicht vom Stichprobenumfang abhängt. Er wird aber in derselben Weise wie Phi interpretiert. Der p-Wert ist eine Kennzahl zur Auswertung von statistischen Tests und wird hier auf einem Signifikanzniveau von $5 \%$ interpretiert, d. h. die Nullhypothese wird verworfen, wenn der p-Wert kleiner oder gleich ist (vgl. Benninghaus 2007: 104-121). Im vorliegenden Zusammenhang wird also nur von einer Fehlerwahrscheinlichkeit ausgegangen. Informationen über tatsächliche Differenzen in der Grundgesamtheit liegen nicht vor, so dass der b-Fehler nicht getestet bzw. bestimmt werden kann. Ausgangspunkt ist also stets eine Nullhypothese, die in der Regel von keinem Zusammenhang oder keinem Unterschied ausgeht. 


\section{Zur Verankerung traditioneller Geschlechterstereotype bei Jugendlichen und Eltern}

Um die Frage zu beantworten, ob eine Verankerung von Geschlechterstereotypen in Werbeanzeigen gegenwärtig noch vorhanden ist und inwiefern von einer Generationenabhängigkeit bei der Inszenierung von geschlechtsspezifischen Zuordnungen gesprochen werden kann, wurden folgende Variablen herangezogen: die beworbenen Produkte, die gezeigten Tätigkeiten und der Goffman'sche entrückte Gesichtsausdruck (vgl. Goffman 1981:256).

Als Produktgruppen, bei denen eine geschlechtsstereotype Unterscheidung angenommen werden kann, wurde die Kosmetikwerbung als Frauenterritorium der Werbung für moderne Medien als typisch technikbasiertem Männerbereich gegenübergestellt. Da die Kosmetikbranche sich durch eine starke Differenziertheit auszeichnet, konnten verschiedene, abgrenzbare Kosmetikproduktgruppen identifiziert werden. Geschlechtsneutrale Körperpflege und -hygiene-Artikel wie Taschentücher und Toilettenpapier wurden so von der Kategorie «verschönernde Kosmetiks abgegrenzt, mit der typisch weibliche Schminkartikel und Stylingutensilien erfasst wurden, welche die Attraktivität zusätzlich steigern. In eine dritte Kosmetikgruppe wurden Produkte gegen inszenierte Defizite eingeordnet. Es handelt sich dabei um Produkte, mit deren Hilfe angebliche Mängel und Defizite, die gesellschaftlich oder zumindest werblich als verpönt gelten, ausgeglichen bzw. beseitigt werden sollen. Hierunter fallen beispielsweise Kosmetika gegen Pigmentstörungen und Altersflecken, Produkte gegen Cellulite, Haarausfall oder Falten.

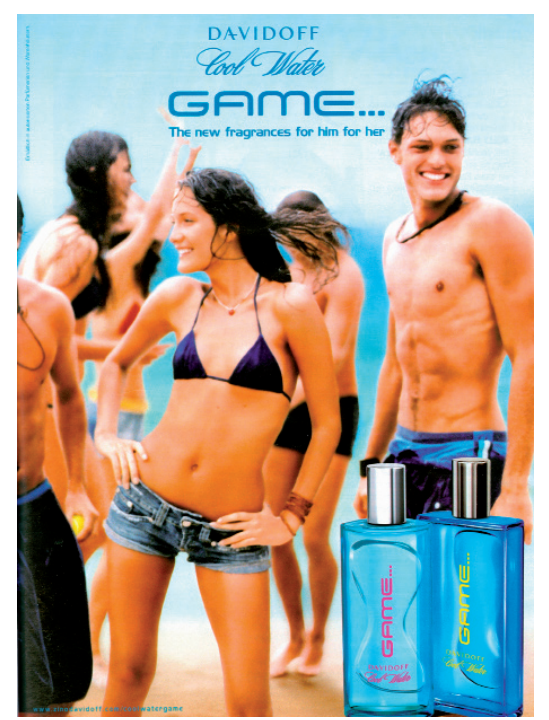

Abb. 2: Werbung für verschönernde Kosmetik (Joy 4/2006: 37) 
Betrachtet man nun die Geschlechterverteilung in den ausgewählten Generationen, so zeigt sich, dass bei den 14- bis 19-Jährigen nicht nur - wie zu erwarten - mit 27 \% ein hoher Anteil der Frauen für verschönernde Kosmetik wirbt, sondern auch junge Männer mit einem Anteil von knapp 20 \% häufig für diese Produktgruppe eingesetzt werden. Hier kann demnach kein signifikanter Unterschied zwischen den Geschlechtern festgestellt werden $(p=.22)$. Interessant erscheint, dass die männlichen Protagonisten vielfach weiblicher Unterstützung «bedürfen` und selten als singuläre Werbeprotagonisten inszeniert werden (Abb. 2). Es könnte sich hierbei um eine Art ‘Eingewöhnung` in diese für das männliche Geschlecht eher ungewohnte Produktsparte handeln.

In der Generation 40-49 fällt dagegen auf, dass der Kosmetikbereich weiterhin ausschliesslich vom weiblichen Geschlecht besetzt ist. Frauen dieser Altersgruppe werben dabei am häufigsten (28,4 \%) für Kosmetika gegen gesellschaftlich inszenierte Defizite, während der Produktbereich bei 40- bis 49-jährigen Männern keine Rolle spielt ( $p=.00, \mathrm{Phi}=.43$ ). Die Thematisierung von Defiziten in der Generation 40-49 scheint sich demzufolge stark auf den weiblichen Körper zu beziehen; männliche Körper werden hingegen weniger streng bewertet. Dieses Ergebnis passt zu der eingangs beschriebenen Werbestrategie, Frauen ab einem gewissen Alter in der Werbung nicht mehr abzubilden bzw. nur dann, wenn sie jünger aussehen (vgl. Eck 2008: 104f). - Ein Ziel, welches es mittels spezieller Kosmetika zu erreichen gilt.

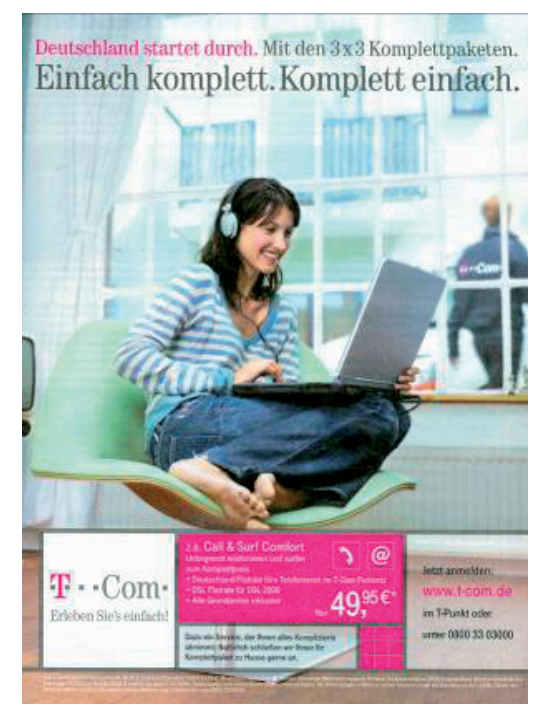

Abb. 3: Junge Frau und moderne Medien (Computer Bild Spiele 11/2006: 13) 
Stellt man diesen Ergebnissen nun die «männliches Produktgruppe der modernen Medien gegenüber, zeigt sich folgendes: Konnte für den Einsatz in der Kosmetikwerbung kein bevorzugtes Geschlecht in der Jugendgeneration festgestellt werden, so wiederholt sich dieses Schema bei der Werbung für moderne Medien. Auch hier ist die Produktsparte bei den 14- bis 19-Jährigen nicht geschlechtsspezifisch determiniert. Junge Frauen werben heute fast genauso häufig wie junge Männer für moderne Medien (Abb. 3), worunter z. B. Handys, EDV-Soft- und Hardware-Komponenten oder MP3-Player zusammengefasst wurden. Die Angleichung betrifft jedoch nicht nur den quantitativen Aspekt, sondern auch die Art der Darstellung. Hier lässt sich keine Differenzierung zwischen einer «typisch männlichens instrumentellen und einer «typisch weiblichen` expressiven Inszenierung feststellen.

Ist das Verhältnis unter den jugendlichen Protagonisten/innen also auch in dieser Produktsparte mit einer Differenz von drei Prozentpunkten (14\% Frauen zu 17 $\%$ Männern) nahezu ausgeglichen ( $p=.55)$, stehen in der Elterngeneration lediglich $6 \%$ Frauen $26 \%$ Männern gegenüber, die hierfür eingesetzt werden ( $p=.00$, $\mathrm{Phi}=.24$ ). Während bei den 40- bis 49-Jährigen der Kosmetikbereich also von Frauen dominiert wurde, wird der Bereich der modernen Medien hauptsächlich mit Männern beworben. Frauen dieser Generation kommen dagegen kaum damit in Berührung.

Bei der Konstruktion der Produktgruppe «moderne Medien` ergaben sich allerdings Schwierigkeiten. Die aktuelle Produktindividualisierung geht einher mit einer Vernetzung und Multifunktionalisierung der Geräte, weshalb man heute auch von Hybridmedien spricht (vgl. dazu auch Krotz 2007: 94ff; Krotz 2008: 55f). Mit einem Handy, ursprünglich als mobiles Telefon gedacht, kann heute z. B. gleichzeitig fotografiert, gefilmt und dank WAP und GPRS im Internet gesurft werden. Computer fungieren zugleich als Unterhaltungsmedium (Musik hören, Fernsehen, Computerspiele spielen, Singstar etc.), Organisator (Terminkalender, Fotoalben, Adressbücher, Geburtstagskalender etc.), Kommunikationsinstrument (E-Mail, Chat etc.), Arbeitsgerät und ebenso als Technikzentrale, in der programmiert und installiert, das Betriebssystem optimiert oder Datenbanken und Informationssysteme analysiert werden. Eine differenzierte Erfassung dieser Produktgruppe ist somit nicht möglich, will man den Anforderungen des Kategoriensystems nach Unabhängigkeit, Trennschärfe und wechselseitiger Exklusivität (vgl. dazu u. a. Merten 1995: 98ff; Früh 1998: 80) gerecht werden. Einschränkend muss somit angemerkt werden, dass die Zusammenfassung dieses Produktbereichs als «moderne Medien` die klassische Zuordnung des technischen Bereichs zum männlichen Geschlecht möglicherweise verzerrt.

Betrachtet man den Bereich der Tätigkeiten, so fällt auf, dass die favorisierte Beschäftigung in der Anzeigenwerbung das Posieren darstellt. Hier zeigen sich für beide Generationen die höchsten Anteilswerte. Die Kategorie wurde immer dann 
codiert, wenn die Personen auf der Anzeige keiner erkennbaren anderen Tätigkeit nachgehen ausser der des Posierens für den Fotografen.

Geschlechtsspezifisch betrachtet erkennt man, dass diese Inszenierungsstrategie bei den Frauen beider Generationen im Schnitt die Hälfte aller Tätigkeiten einnimmt (bei 14- bis 19-Jährigen $47 \%$, bei 40- bis 49-Jährigen $58 \%$; $p=.11$ ). Aber auch Männer in der Werbung posieren häufig. So dominiert diese Tätigkeit ebenso beim männlichen Geschlecht der Generation 40-49 (Abb. 4). Von den männlichen Jugendlichen posieren immerhin rund ein Viertel $(p=.57)$. Es handelt sich bei dieser Personengruppe damit um die zweithäufigste Beschäftigungsart. An erster Stelle steht das gesellige Zusammensein mit Anderen.

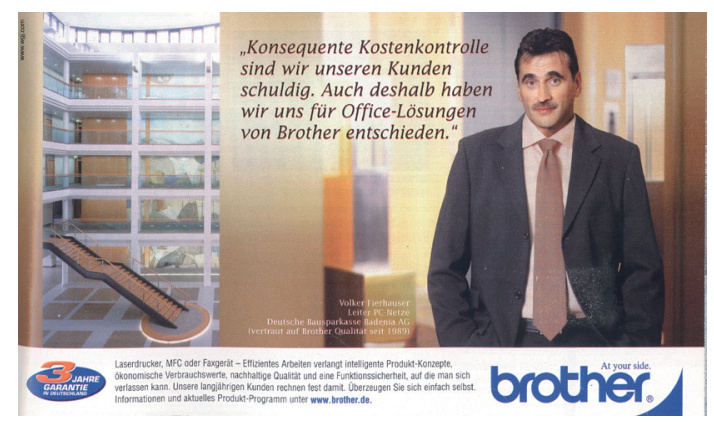

Abb. 4: Posierender Mann der Generation 40-49 (Focus 19/2004: 125)

Generationen- und geschlechtsübergreifend wird das Gesellige (Gespräche, Flirten, Nutzung von Kommunikationsmitteln, Beschäftigung mit Kindern etc.) als zweithäufigster Tätigkeitsbereich verwendet, gefolgt von Menschen in Aktion (Sport, Ausübung von Hobbys, Urlaub etc.) oder beim Müssiggang (Lesen, Fernsehen, Musik hören, allgemeine Entspannung, Körperpflege etc.). Interessant im Vergleich zu vorherigen Untersuchungsergebnissen (vgl. Schmerl 1994: 139ff; Bergler u. a. 1992: 32ff) erscheint hierbei, dass bei Männern und Frauen der Generation 40-49 kein signifikanter Unterschied bei der Kategorie 〈Aktionismus〉 besteht $(p=.61)$, während Tätigkeiten aus der Kategorie «Musse», worunter eher passive, selbstbezogene Tätigkeiten gefasst wurden, bei Männern wesentlich stärker ins Gewicht fallen ( $p=.04, P h i=.15)$. Ein Bild, das nicht zum klassischen, aktiven Mann der Werbung passt (vgl. Krohne 1995; Zurstiege 1998). 


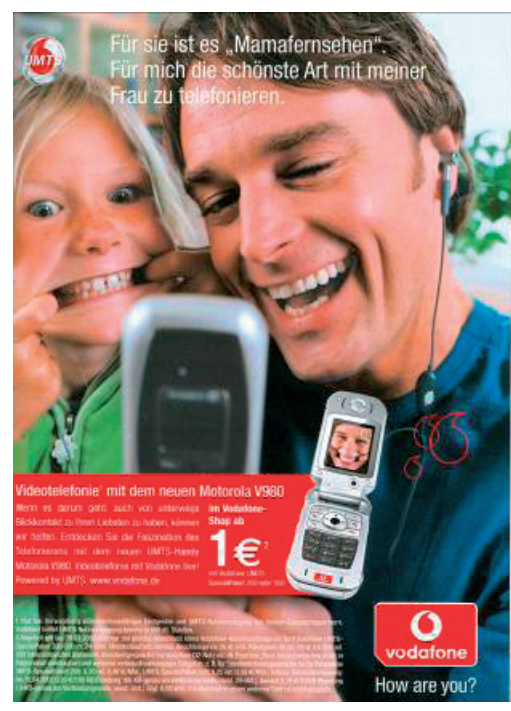

Abb. 5: Vater mit Kind (Joy 01/2005: 65)

Bezüglich der Tätigkeiten fällt speziell bei der Generation 40-49 zusätzlich ein ambivalenter Aspekt auf: Einerseits sind Männer dieser Altersgruppe gemäss klassischer Vorstellungen weitaus stärker im Beruf vertreten als Frauen. Andererseits ist es jedoch auch das männliche Geschlecht, das häufiger in traditionell weiblichen Bereichen wie dem Haushalt oder bei der Beschäftigung mit Kindern abgebildet wird (Abb. 5).

Die Verteilung der Tätigkeiten unter den Geschlechtern insgesamt zeigt: Männliche Tätigkeitsfelder sind vielfältiger und variationsreicher als weibliche. Männer gehen häufiger einem Beruf nach, befinden sich in geselligen bzw. sozialen Situationen, üben aktionale Tätigkeiten aus oder lassen sich bei passiven, selbstbezogenen Tätigkeiten treiben. Demgegenüber stehen nahezu die Hälfte aller weiblichen Akteure, die ausschliesslich posieren. Tätigkeiten von Frauen erscheinen folglich weniger zielgerichtet. Hinzugefügt werden muss jedoch, dass aufgrund des relativ hohen Anteils männlicher Posierender und der ähnlichen geschlechtlichen Verteilung der aktiven und müssigen Personen die gängige Assoziation des weiblichen Geschlechts mit Passivität und des männlichen Geschlechts mit Aktivität an dieser Stelle nicht bestätigt werden kann.

Zieht man zur Beantwortung der Forschungsfrage den Gesichtsausdruck als mögliches geschlechtsdeterminiertes Merkmal hinzu, fällt auf, dass unabhängig von Geschlecht und Alter der glückliche Gesichtsausdruck (Lachen) überwiegt. Weiter zeigt sich, dass auffallend viele Jugendliche entrückt blicken, d. h. ihren Blick ziellos in die Ferne schweifen lassen, abwesend wirken. Goffman definiert diesen Gesichtsausdruck auch als ein «Treibenlassen vor Anker» (Goffman 1981: 256), der in erster Linie bei der Inszenierung von weiblichen Protagonisten seine Anwendung 
fand. Interessant ist, dass dieser ehemals feminine Blick in der hier vorliegenden Analyse bei beiden Geschlechtern der Generation 14-19 etwa gleich stark vertreten ist (ca. $14 \% ; p=.70 ; A b b .6$ ), bei der Generation 40-49 dagegen bei beiden Geschlechtern keine Rolle spielt.

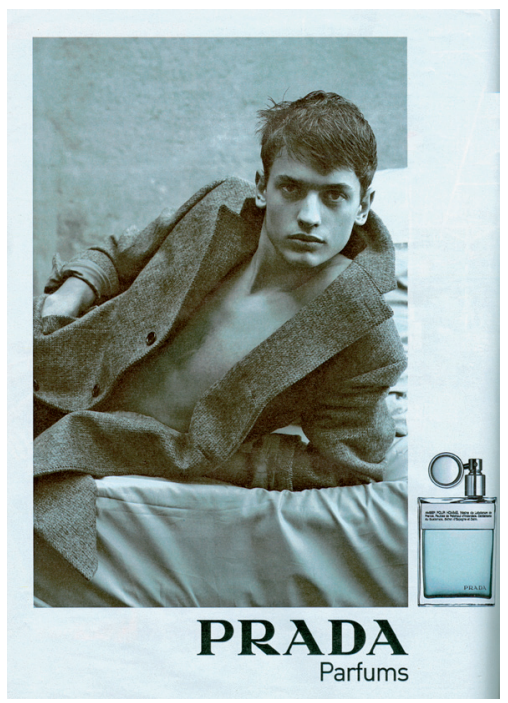

Abb. 6: Junger Mann mit entrücktem Blick (Stern 43/2006: 24)

Um die Realitätsnähe der Darstellungen zu kategorisieren, wurde bei der Variable 〈Produkt-Person-Beziehung〉 von einer realitätsnahen zu einer realitätsfernen Beziehung zwischen der dargestellten Person und dem gezeigten Produkt abgestuft. Als produktunabhängige/r Akteur/in oder Produktnutzer/in agiert die Person in einem Handlungsraum und bewegt sich in einer wirklichkeitsgetreuen Szene. Produktpräsentanten/innen erscheinen als Mittelkategorie zwischen realen und realitätsfernen Beziehungen. Hier präsentiert die Person (meist durch Posieren) das Produkt, z. B. Kleidung oder frisch gefärbte Haare. Die Person kann sich dabei durchaus in einem Handlungsraum befinden, die Szene wirkt jedoch gestellt. Ist die Szene noch stärker konstruiert und komplett aus einem realen Handlungsraum herausgenommen, wurden die Akteure und Akteurinnen als Vorführobjekt codiert, an dem z. B. eine bestimmte Creme erfolgreich angewandt wurde. Ausserdem konnte die Person als Testimonial auftreten oder als reines Dekorationsobjekt ohne funktionale Beziehung zum Produkt inszeniert werden.

Insgesamt beträgt das Verhältnis der realitätsnäheren Darstellungsformen (‘produktunabhängige/r Akteur/in`, ‘aktuelle/r Produktnutzer/in) und «potentielle/r Produktnutzer/ins) etwa 60 \% zu knapp $40 \%$ realitätsferneren Darstellungsformen (〈Produktpräsentant/in〉, 〈Vorführobjekt〉, 〈Dekorationsobjekt〉). Dabei fällt auf, dass 75 \% der Männer in einem realen Produkt-Person-Zusammenhang dargestellt wer- 
den, aber nur die Hälfte der Frauen. Die Präsentation in einer realitätsnahen Produkt-Person-Beziehung scheint demzufolge eindeutig eine Inszenierungsstrategie für das männliche Geschlecht zu sein $(p=.00$, Phi=.26), während Frauen signifikant häufiger in einem realitätsfernen Kontext erscheinen $(p=.00$, Phi $=.28)$. Das gilt sowohl für die Generation 14-19 als auch für die 40- bis 49-Jährigen.

Als nächstes geht es um die Problematik der Schönheitsideale. Dabei wird davon ausgegangen, dass Menschen sich im Allgemeinen an Ihresgleichen orientieren, also Werbeprotagonisten/innen ihrer eigenen Altersgruppe besondere Aufmerksamkeit schenken (vgl. Bergler 1965: 112; Packard 1969: 66; Zurstiege 1998: 146, 185). Wie bereits im ersten Teil dieses Beitrags diskutiert, können mediale Ideale speziell für Heranwachsende im Zuge der Sozialisation eine Gefahr darstellen. Der Generationenaspekt steht deshalb auch bei der zweiten Forschungsfrage im Vordergrund.

\section{Idealisierende Darstellung der Generationen}

Zur Analyse der Idealisierung wurde eine Reihe von äusserlichen Erscheinungsmerkmalen erhoben, anhand derer verglichen werden kann, wie ausgedehnt genormte Schönheitsideale und Attraktivitätsvorstellungen in der Werbung ihren Einsatz finden.

Die Grundlage für den generellen Vergleich bildet die Unterscheidung zwischen einer natürlichen und einer makellosen Erscheinungsweise. Ein natürliches Erscheinungsbild wurde dann codiert, wenn einem die Person prinzipiell häufiger auf der Strasse begegnen könnte wie beispielsweise die Darsteller/innen auf den folgenden Anzeigen (Abb. 7 und 8).

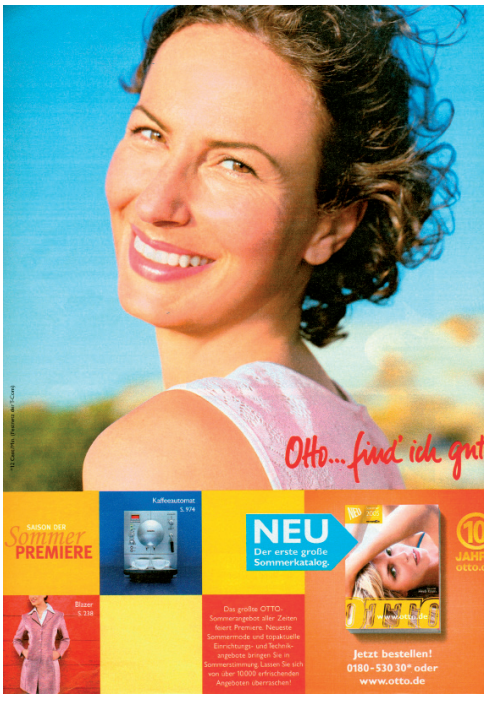

Abb. 7: Natürliche Frau (Stern 21/2005: 12)

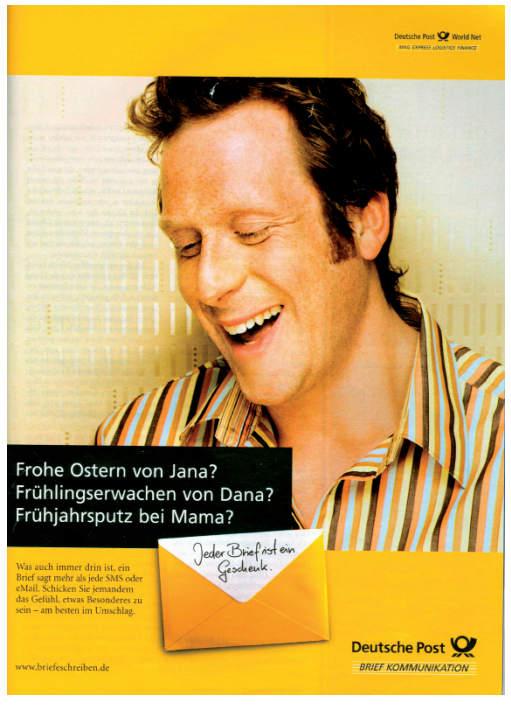

Abb. 8: Natürlicher Mann (Brigitte 8/2004: 99) 
Anzeigen mit natürlichen Personen zeichnen sich dadurch aus, dass nicht alles restlos retuschiert wird, sondern kleine Abweichungen vom Schönheitsideal (z. B. Falten, Zahnlücke, fülligere Figur, dünnes Haar, Sommersprossen o. ä.) erhalten bleiben

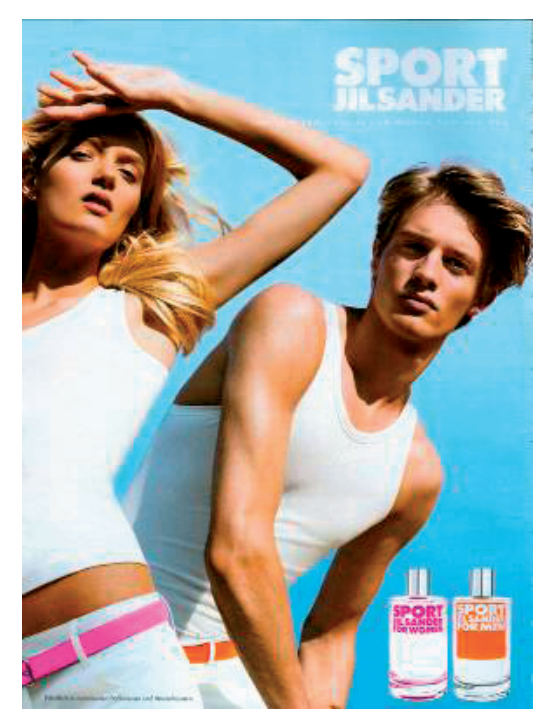

Abb. 9: Makellose Jugendliche (Joy 10/2005: 2)

Als makellos wurde das Aussehen hingegen eingestuft, wenn es sich um Personen handelt, die vollkommen dem medial bevorzugten Schönheitsideal entsprechen und damit fast schon realitätsfern bzw. künstlich wirken (Abb. 9). Als Indikatoren wurden hier das gemeinsame Auftreten der Merkmale reine Haut, Faltenlosigkeit, strahlender Teint, glänzende Haare und Schlankheit festgelegt.

Natürliche Darstellungen dominieren bei beiden Generationen, die Generation 40-49 wird allerdings signifikant häufiger mit einem natürlichen Aussehen präsentiert (63,6 \% und 93,7\%; $p=.00$, Phi=.35), während die 14- bis 19-Jährigen signifikant häufiger makellos inszeniert werden (32\% zu 4,2\%; $p=.00$, Phi $=.34$ ).

Besonders die Schlankheit rückt oft ins Zentrum der Kritik medialer Darstellungen. Vorwürfe richten sich gegen ein propagiertes Körperideal, das einer «krankhaften Untergewichtigkeit entspricht» (Kröner-Herwig 2001: 135) und damit den Typus der Barbiepuppe - «vom Schlankheitswahn ausgemergelt, aber ewig lächelnd» (MühIen Achs 1990: 97) - abbildet. Gerade diese medial propagierten Körperformen werden als Mitauslöser für Schlankheitswahn, Diäthalten, Hungern und Unzufriedenheit mit dem eigenen Körper im Jugendalter angesehen.

Die hier vorliegende Analyse kann die Begrenztheit der Körperformen im werblichen Kontext bestätigen: Jugendliche erscheinen auf Werbeanzeigen ausschliesslich dünn oder schlank, wobei keine bedeutsamen Differenzen zwischen den Ge- 
schlechtern herausgestellt werden können ( $p=.42)$. Es findet in dieser Generation somit eine starke einseitige Normierung auf den schlanken oder sehr schlanken Körper statt $(\mathrm{p}=.00, \mathrm{Phi}=.27)$.

Auch bei der Generation 40-49 dominiert der schlanke Körper eindeutig geschlechtsübergreifend $(p=.00, P h i=.20)$. Allerdings findet sich hier zumindest die Tendenz, Werbeprotagonisten/innen ebenfalls mit vom Schlankheitsmodell abweichenden Figuren abzubilden.

Die werbliche Normierung und Typisierung, die bei jugendlichen Werbedarsteller/innen besonders stark zum Ausdruck kommt, kann durch weitere äusserliche Erscheinungsmerkmale verdeutlicht werden. Entsprechend der klassischen Geschlechterstereotype haben knapp 70 \% der jungen Frauen lange Haare, während junge Männer sich zu knapp 80 \% mit einem Kurzhaarschnitt präsentieren. Bei Männern der Generation 40-49 dominieren ebenfalls die kurzen Haare; es findet sich somit kein signifikanter Unterschied zwischen den männlichen Generationen $(p=.61)$. Dem weiblichen Geschlecht der Generation 40-49 wird hingegen mehr Spielraum bei der Frisur gestattet. Hier ist die Anzahl derer, die mit langen (31\%), mit mittellangen (37\%) und kurzen Haaren (21\%) inszeniert werden, nahezu ausgeglichen. Es zeigt sich ausserdem, dass Frauen beider Generationen zwar meistens sichtbar geschminkt gezeigt werden (ca. $70 \%$ ), jugendliche Frauen allerdings häufiger sehr auffällig geschminkt (12,8 \% zu 4,5\%) in der Werbung präsentiert werden, auch wenn diese Differenz sich statistisch an der Signifikanzgrenze bewegt $(p=.06)$.

\section{Der Apfel fällt nicht weit vom Stamm?}

Es gibt keine Klassifikation der Menschen, die so fest in der Menschwerdung verwurzelt ist und die eine Gesellschaft und das Zusammenleben der Menschen so fundamental und nachhaltig strukturiert, reguliert und ordnet, wie die Unterscheidung in männlich und weiblich (vgl. zu den folgenden Ausführungen auch Derra 2006: 10-15).

Die Geschlechtszugehörigkeit äussert sich in den unterschiedlichsten Kontexten: in der Familie und in öffentlichen Räumen, in der alltäglichen Interaktion und der medialen Kommunikation, in der Schule und im Beruf, in der Freizeit sowie in ökonomischen und politischen Diskursen, in der Sexualität wie in der Religion. Budde (kursiv i. Org., 2003: 17) formuliert hierzu treffend: «jede soziale Situation ist auch immer geschlechtlich eingefärbt, sozusagen 'gegendertı.»

Neben dem Alter und der ethnischen Zugehörigkeit, bei denen allerdings bis zu einem gewissen Grad Fehleinschätzungen erlaubt sind, ist das Geschlecht einer Person ein unmittelbar wahrgenommenes Merkmal, das eine erste automatische Kategorisierung zwischen Personen auslöst. Das Geschlecht fungiert somit als «wirkungsvollste Identifikations-Schranke» in der Gesellschaft (Mühlen Achs 1990: 89). 
Dass fundamentale Unterschiede zwischen den Geschlechtern herrschen, gehört zu den «fraglosen und nicht weiter begründungsbedürftigen Selbstverständlichkeiten unseres Alltagswissens» (Wetterer 2004: 122). Die ursprünglich mit der Kategorie Geschlecht verknüpften Aspekte der Unveränderbarkeit, Lebenslänglichkeit, Konstanz und der Totalinklusion, dass jeder jederzeit entweder männlich oder weiblich sein muss, also der einen oder anderen Kategorie angehörig (vgl. dazu Tyrell 1986: 464ff), sind jedoch auf wissenschaftlicher Ebene ins Wanken geraten und dürfen keine uneingeschränkte Gültigkeit mehr für sich beanspruchen.

Die feministische Seite ist es, in der erstmals Stimmen laut werden, sich gänzlich von der Debatte um die Determinierung durch primäre, sekundäre und tertiäre Geschlechtsmerkmale (Geschlechtsorgane, -drüsen, -hormone, Morphologie) abzuwenden und das Geschlecht in seiner Gesamtheit als vollständig konstruiert zu erfassen (Hagemann-White 1984: «kulturelles System der Zweigeschlechtlichkeit»).

Die Geschlechtsidentität, die sich als Wahrnehmung, Erfahrung und das Erleben der eigenen Geschlechtszugehörigkeit von aussen sowie die blosse Selbstzuschreibung als männlich und weiblich definiert (vgl. Degenhardt \& Trautner 1979: 14; Behnke \& Meuser 1999: 52), wurde auch von Goffmans Einwurf, "dass es so etwas wie eine Geschlechtsidentität nicht gibt» (Goffman 1981: 37), hinterfragt und die Grenzziehung der Geschlechterverhältnisse relativiert. Nach Goffman besitzen Menschen von Natur aus nur die Eigenschaft, «Abbilder von Männlichkeit und Weiblichkeit entwerfen und verstehen zu lernen» (Goffman 1981: 37) und verfügen über diese Eigenschaft eben als Personen und nicht als Mann oder Frau. Diese Abbilder, die Goffman als «Prototypen des essentiellen Ausdrucks» (Goffman 1981: 34) bezeichnet, gehören bei ihm noch zu der «elementarsten Charakterisierung eines Menschen» (ebd.) und stellen das Medium der Verständigung über die Identität eines Individuums dar. Dagegen kann knapp 25 Jahre später Meuser - allerdings nicht unkritisch - keine entsprechende 〈Hommage〉 an die Geschlechtszugehörigkeit mehr offerieren: «Die Begriffe Mann und Frau bezeichnen keine sozial bedeutsamen Kategorien mehr. Die Sozialordnung der Zweigeschlechtlichkeit wird dekonstruiert (...). Alles erscheint möglich» (Meuser 2000: 217). Elementar ist hier die Unterscheidung zwischen dem anerkannten Gedanken der Dekonstruktion der Kategorie «Geschlecht» auf der sozialen Ebene (gender) und der stark kritisierten Annahme einer vollständig, also bis hin zur biologischen Geschlechtskomponente (sex) konstruierten Geschlechtskategorie.

Eine Aufhebung der Geschlechtlichkeit auf der äusserlichen Ebene kann in der aktuellen werblichen Geschlechterinszenierung nicht festgestellt werden; so erscheinen beispielsweise junge Frauen typischerweise mit langen Haaren, junge Männer mit kurzen. Bestätigende Hinweise zur behaupteten soziokulturellen Dekonstruktion der Zweigeschlechtlichkeit finden sich jedoch in Form einer Vermischung ehemals geschlechtlich getrennter Bereiche vor allem bei den Jugendlichen, wie 
die Ergebnisse der Produktarten 〈Kosmetik〉 und «moderne Medien〉, des ‘Aussehens〉, des ‘Gesichtsausdrucks〉 und der Tätigkeit 〈Posieren〉 gezeigt haben. Auch der aktuelle gesellschaftliche, immer wieder kritisierte Körperkult kommt bei der jugendlichen Generation beider Geschlechter gleichermassen zum Ausdruck. Das verdeutlichen die Ergebnisse der Variablen des äusserlichen Erscheinungsbildes: 〈Aussehen〉, 〈Figur〉, ¿Frisur〉 und, für das weibliche Geschlecht geltend, die «Schminkes. So erscheinen junge Frauen genau wie Männer ihrer Generation schlank und schön. Zusätzlich verstärkt wird dies durch die starke Fixierung der Altersgruppe auf den Produktbereich der «verschönernden Kosmetik».

Bei der Generation 40-49 gibt es dagegen neben innovativen Inszenierungsformen stärker auch traditionell geschlechtsspezifische Merkmale. So werden bei der Beschäftigung mit der Familie, im Haushalt, beim Posieren und bei der Inszenierung des äusserlichen Erscheinungsbildes fortschrittliche Darstellungsarten verwendet. Die Produktgruppen Kosmetik und moderne Medien, die Ausübung einer beruflichen Tätigkeit sowie der gezeigte Gesichtsausdruck weisen stattdessen eine weiterhin traditionelle Verhaftung auf.

Die Werbeprotagonisten/innen der Generation 40-49 - und dort besonders die Männer - besitzen im Gegensatz zu den Vertretern/innen der Jugendgeneration körperliche Freiräume, was ihr Aussehen, ihre Figur und ihre Frisur betrifft. Hier wird zumindest ansatzweise eine realitätsnähere Darstellung genutzt und damit nicht ausschliesslich ein einseitig normiertes Personenideal präsentiert - durchaus eine zu begrüssende Entwicklung.

Die Ausgangsfrage «Der Apfel fällt nicht weit vom Stamm?` kann somit beantwortet werden: Es lassen sich signifikante Differenzen in den Darstellungsschemata der Jugend- und Elterngeneration ausmachen. Innerhalb der einzelnen Altersgruppen kann allerdings von einer ambivalent zu bewertenden Angleichung der Geschlechter gesprochen werden. Besonders die werbliche Zentrierung gerade der heranwachsenden Generation auf Körperlichkeit und Schönheit betrifft nun beide Geschlechter. Dies birgt im Zuge der Diskussion medialer Orientierungs- und Leitbilder das bereits beschriebene Gefahrenpotential (z. B. Magersucht, übersteigerter Fitnesswahn, Körper als Fetisch). Dadurch wird fraglich, ob die Auflösung der häufig kritisierten Geschlechterstereotype, wie bereits vielfach beschrieben (vgl. z. B. Bock 2004), ausschliesslich positiv konnotiert werden kann. Vielmehr erscheint eine Differenzierung zwischen dem Gewinn von Freiheit und Vielseitigkeit durch die Lösung von Stereotypen einerseits und der möglichen Ausweitung von Normierungen und einseitigen Typisierungen bzw. Idealisierungen andererseits für die weitere wissenschaftliche Auseinandersetzung mit dieser Thematik zwingend notwendig. 


\section{Literaturverzeichnis}

Angerer, Marie-Luise; Dorer, Johanna (Hrsg.) (1994). Gender und Medien: theoretische Ansätze, empirische Befunde und Praxis der Massenkommunikation. Ein Textbuch zur Einführung. Wien: Braumüller.

Baacke, Dieter u. a. (1993). Kinder und Werbung. Stuttgart, Berlin, Köln: Kohlhammer.

Baacke, Dieter u. a. (1999). Zielgruppe Kind: Kindliche Lebenswelt und Werbeinszenierungen. Opladen: Leske + Budrich.

Becker, Ruth; Kortendiek, Beate (Hrsg.) (2004). Handbuch Frauen- und Geschlechterforschung. Theorien, Methoden, Empirie. Wiesbaden: VS-Verlag.

Behnke, Cornelia; Meuser, Michael (1999). Geschlechterforschung und qualitative Methoden. Opladen: Leske + Budrich.

Benninghaus, Hans (2007). Deskriptive Statistik. Eine Einführung für Sozialwissenschaftler. 11. Aufl. Wiesbaden: VS-Verlag.

Bergler, Georg (1965). Werbung und Gesellschaft. Essen: Giradet.

Bergler, Reinhold u. a. (1992). Frau und Werbung: Vorurteile und Forschungsergebnisse. Köln: Deutscher Instituts-Verlag.

Bieber-Delfosse, Gabrielle (2002). Vom Medienkind zum Kinderstar. Einfluss- und Wirkfaktoren auf Vorstellungen und Prozesse des Erwachsenwerdens. Opladen: Leske + Budrich.

Bock, Ulla (2004). «Androgynie. Von Einheit und Vollkommenheit zu Vielfalt und Differenz.» Handbuch Frauen- und Geschlechterforschung. Theorien, Methoden, Empirie. Hrsg. von Ruth Becker u. Beate Kortendiek. Wiesbaden: VS-Verlag. 99-103.

Budde, Jürgen (2003). «Die Geschlechterkonstruktion in der Moderne. Einführende Betrachtungen zu einem sozialwissenschaftlichen Phänomen». Medien. Sozialisation. Geschlecht. Fallstudien aus der sozialwissenschaftlichen Forschungspraxis. Hrsg. von Renate Luca. München: kopaed. 11-25.

Bundeszentrale für gesundheitliche Aufklärung (2008): Essstörungen/Information für Betroffene, Angehörige, Fachleute und allgemein Interessierte: http://www. bzga-essstoerungen.de/allgemeine-infos/zu_dick_oder_zu_duenn/schoenheitsideale.htm (03.04.2008).

Bundeszentrale für politische Bildung (Hrsg.) (1992). Frauenbilder im Fernsehen. Beiträge und Materialien einer Fachtagung vom 25. bis 27. August 1991 in Augsburg. Bonn: Bundeszentrale für politische Bildung.

Degenhardt, Annette; Trautner, Hanns Martin (1979). «Einleitung.» Geschlechtstypisches Verhalten: Mann und Frau in psychologischer Sicht. Hrsg. von Annette Degenhardt u. Hanns Martin Trautner. München: Beck. 1-25.

Degenhardt, Annette; Trautner, Hanns Martin (Hrsg.) (1979). Geschlechtstypisches Verhalten: Mann und Frau in psychologischer Sicht. München: Beck. 
Derra, Julia Maria (2006). "Darf ich auch einmal irgendwo nicht reinpassen? - Du darfst!» Eine inhaltsanalytische Untersuchung von Frauendarstellungen in Anzeigenwerbung ausgewählter Zeitschriften mit anschliessender Befragung einer entsprechenden Rezipientengruppe. Trier: Unveröffentlichte Magisterarbeit.

Derra, Julia Maria; Jäckel, Michael (2009). «¿Darf ich auch einmal irgendwo nicht reinpassen?> Darstellung und Wahrnehmung von Frauen in Werbeanzeigen.» Theatralisierung der Gesellschaft. Bd. 2: Medientheatralität und Medientheatralisierung. Hrsg. von Herbert Willems. Wiesbaden: VS-Verlag (im Erscheinen).

Eck, Cornelia (2008). Schattenbilder - Männlichkeit und Weiblichkeit in Werbeanzeigen. Hamburg: Verlag Dr. Kova .

Eck, Cornelia; Jäckel, Michael (2009). "Werbung mit dem kleinen Unterschied.» Theatralisierung der Gesellschaft. Bd. 2: Medientheatralität und Medientheatralisierung. Hrsg. von Herbert Willems. Wiesbaden: VS-Verlag (im Erscheinen).

Ferchhoff, Wilfried (1999). Jugend an der Wende vom 20. zum 21. Jahrhundert. Lebensformen und Lebensstile. 2., überarb. u. akt. Aufl. Opladen: Leske + Budrich.

Forster, Edgar J. (1995). «Die unsichtbare Allgegenwart des Männlichen in den Medien.» Geschlecht und Medien. Hrsg. von Gitta Mühlen Achs u. Bernd Schorb. München: kopaed. 57-69.

Franz, Carmen; Schwibbe, Gudrun (Hrsg.) (2001). Geschlecht weiblich: Körpererfahrungen - Körperkonzepte. Berlin: Edition Ebersbach.

Früh, Werner (1998). Inhaltsanalyse. Theorie und Praxis. 4., überarb. Aufl. Konstanz: UVK Medien.

Gaugele, Elke; Reiss, Kristina (Hrsg.) (2003). Jugend, Mode, Geschlecht. Die Inszenierung des Körpers in der Konsumkultur. Frankfurt/Main, New York: Campus.

Geulen, Dieter (2007). «Sozialisation.» Lehrbuch der Soziologie. Hrsg. von Hans Joas. 3, überarb. u. erw. Aufl. Frankfurt/Main, New York: Campus. 138-158.

Göttlich, Udo u. a. (Hrsg.) (1998). Kommunikation im Wandel. Zur Theatralität der Medien. Köln: Halem.

Goffman, Erving (1981). Geschlecht und Werbung. Frankfurt/Main: suhrkamp. (Original 1976: Gender Advertisements. New York: Harper \& Row).

Hagemann-White, Carol (1984). Sozialisation: Weiblich - männlich? Opladen: Leske und Budrich (Alltag und Biografie von Mädchen, Bd. 1).

Hastenteufel, Regina (1980). Das Bild von Mann und Frau in der Werbung. Eine Inhaltsanalyse zur Geschlechtsspezifität der Menschendarstellung in der Anzeigenwerbung ausgewählter Zeitschriften unter besonderer Berücksichtigung des alten Menschen. Bonn: Univ. Diss. 
Hettlage, Robert; Vogt, Ludgera (Hrsg.) (2000). Identitäten in der modernen Welt. Wiesbaden: Westdeutscher Verlag.

Hillmann, Karl-Heinz (2007). Wörterbuch der Soziologie. 5., vollst. überarb. u. erw. Aufl. Stuttgart: Kröner.

IVW (Hrsg.) (2006). Auflagenliste 1/2006: www.ivw.de (03.07.2006).

Jäckel, Michael (2009). «Ältere Menschen in der Werbung.» Medien im höheren Lebensalter. Hrsg. von Bernd Schorb, Anja Hartung u. Wolfgang Reissmann. Wiesbaden: VS-Verlag (im Erscheinen).

Joas, Hans (Hrsg.) (2007). Lehrbuch der Soziologie. 3., überarb. u. erw. Aufl. Frankfurt/Main, New York: Campus.

Kleinschmidt, Anna (2003). «Körperlos im Körperwahn.» Jugend, Mode, Geschlecht. Die Inszenierung des Körpers in der Konsumkultur. Hrsg. von Elke Gaugele u. Kristina Reiss. Frankfurt/Main, New York: Campus. 95-112.

Körber, Christian; Schaffar, Andrea (2003). «ldentitätskonstruktionen in der Mediengesellschaft. Theoretische Annäherungen und empirische Befunde.» Medien-Impulse. Nr. 09. 80-86.

Kröner-Herwig, Birgit (2001). «Der Körper als Subjekt und Objekt. Zusammenhänge mit Störungen des Befindens, Erlebens und Verhaltens.» Geschlecht weiblich: Körpererfahrungen - Körperkonzepte. Hrsg. von Carmen Franz u. Gudrun Schwibbe. Berlin: Edition Ebersbach. 133-147.

Krohne, Stefan (1995). «It's a Men's World. Männlichkeitsklischees in der deutschen Fernsehwerbung.» Werbung, Medien und Kultur. Hrsg. von Siegfried J. Schmidt u. Brigitte Spiess. Opladen: Westdeutscher Verlag. 136-152.

Krotz, Friedrich (2003). «Medien als Ressource der Konstitution von Identität. Eine konzeptionelle Klärung auf der Basis des Symbolischen Interaktionismus.» Medienidentitäten. Identität im Kontext von Globalisierung und Medienkultur. Hrsg. von Carsten Winter u. a. Köln: Halem. 27-48.

Krotz, Friedrich (2007). Mediatisierung: Fallstudien zum Wandel von Kommunikation. Wiesbaden: VS-Verlag

Krotz, Friedrich (2008). «Kultureller und gesellschaftlicher Wandel im Kontext des Wandels von Medien und Kommunikation.» Medienkultur und soziales Handeln. Hrsg. von Tanja Thomas. Wiesbaden: VS-Verlag. 43-62.

Luca, Renate (2003). «Mediensozialisation. Weiblichkeits- und Männlichkeitsentwürfe in der Adoleszenz.» Medien. Sozialisation. Geschlecht. Fallstudien aus der sozialwissenschaftlichen Forschungspraxis. Hrsg. von Renate Luca. München: kopaed. 39-54.

Luca, Renate (Hrsg.) (2003). Medien. Sozialisation. Geschlecht. Fallstudien aus der sozialwissenschaftlichen Forschungspraxis. München: kopaed.

Luhmann, Niklas (2004). Die Realität der Massenmedien. 3. Aufl. Wiesbaden: VSVerlag. 
Mediaanalyse (2006): Onlineanalyse. Pressemedien I - Burda Advertising Center GmbH: http://ma.bik-gmbh.de/burda/ (03.07.2006).

Merten, Klaus u. a. (Hrsg.) (1994). Die Wirklichkeit der Medien. Eine Einführung in die Kommunikationswissenschaft. Opladen: Westdeutscher Verlag.

Merten, Klaus (1995). Inhaltsanalyse. Einführung in Theorie, Methode und Praxis. 2. verb. Aufl. Opladen: Westdeutscher Verlag.

Meuser, Michael (2000). «Entgrenzte Geschlechterverhältnisse? Enttraditionalisierung und habituelle Rahmen.» Identitäten in der modernen Welt. Hrsg. von Robert Hettlage u. Ludgera Vogt. Wiesbaden: Westdeutscher Verlag. 217-238.

Mikos, Lothar (2005). «Aufwachsen in mediatisierten Lebenswelten. Medien-Identität-Identifikationen.» tv diskurs - Verantwortung in audiovisuellen Medien. $\mathrm{H}$. 34. 20-25.

Mühlen Achs, Gitta (1990). "Von Männern und Mäuschen. Zur psychologischen Funktion männlicher und weiblicher Rollen in Film und Fernsehen.» Bildersturm. Frauen in den Medien. Hrsg. von Gitta Mühlen Achs. München: Frauenoffensive. 88-106.

Mühlen Achs, Gitta (Hrsg.) (1990). Bildersturm. Frauen in den Medien. München: Frauenoffensive.

Mühlen Achs, Gitta (1995). «Frauenbilder: Konstruktionen des anderen Geschlechts.» Geschlecht und Medien. Hrsg. von Gitta Mühlen Achs u. Bernd Schorb. München: kopaed. 13-37.

Mühlen Achs, Gitta; Schorb, Bernd (Hrsg.) (1995). Geschlecht und Medien. München: kopaed.

Oerter, Rolf; Dreher, Eva (1998). «Jugendalter.» Entwicklungspsychologie. Hrsg. von Rolf Oerter u. Leo Montada. 4. korr. Aufl. Weinheim: Psychologie Verlags Union. 310-395.

Oerter, Rolf; Montada, Leo (Hrsg.) (1998). Entwicklungspsychologie. 4. korr. Aufl. Weinheim: Psychologie Verlags Union.

Packard, Vance (1969). Die geheimen Verführer. Der Griff nach dem Unbewussten in Jedermann. Düsseldorf, Wien: Econ.

Röhr-Sendlmeier, Una; Ueing, Sarah (2004). «Das Altersbild in der Anzeigenwerbung im zeitlichen Wandel.» Zeitschrift für Gerontologie und Geriatrie. Vol. 37. Nr. 1. 56-62.

Schmerl, Christiane (1984). Das Frauen- und Mädchenbild in den Medien. Opladen: Leske + Budrich.

Schmerl, Christiane (1994). "Die schönen Leichen aus Chromdioxyd und aus Papier: Frauenbilder in der Werbung.» Gender und Medien: theoretische Ansätze, empirische Befunde und Praxis der Massenkommunikation. Ein Textbuch zur Einführung. Hrsg. von Marie-Luise Angerer u. Johanna Dorer. Wien: Braumüller. 134-151. 
Schmidt, Siegfried J.; Spiess, Brigitte (Hrsg.) (1995). Werbung, Medien und Kultur. Opladen: Westdeutscher Verlag.

Schmidt, Siegfried J. (2000). Kalte Faszination. Medien, Kultur, Wissenschaft in der Mediengesellschaft. Weilerswist: Velbrück.

Schorb, Bernd (2006). «Identitätsbildung in der konvergenten Medienwelt.» Neue Wege durch die konvergente Medienwelt. Studie im Auftrag der Bayrischen Landeszentrale für neue Medien (BLM). Hrsg. von Ulrike Wagner u. Helga Theunert. München: Reinhard Fischer. 149-160.

Schorb, Bernd; Hartung, Anja; Reissmann, Wolfgang (Hrsg.) (2009). Medien im höheren Lebensalter. Wiesbaden: VS-Verlag (im Erscheinen).

Sowinski, Bernhard (1998). Werbung. Tübingen: Niemeyer.

Spiess, Brigitte (1992). «Frauenbilder in der Fernseh-Werbung. Gefangen zwischen alten Leitbildern und neuen Rollenvorstellungen.» Frauenbilder im Fernsehen. Beiträge und Materialien einer Fachtagung vom 25. bis 27. August 1991 in Augsburg. Hrsg. von Bundeszentrale für politische Bildung. Bonn: Bundeszentrale für politische Bildung. 91-108.

Spiess, Brigitte (1994). «Weiblichkeitsklischees in der Fernsehwerbung.» Die Wirklichkeit der Medien. Eine Einführung in die Kommunikationswissenschaft. Hrsg. von Klaus Merten u. a. Opladen: Westdeutscher Verlag. 408-426.

Statistisches Bundesamt (Hrsg.) (2007). Geburten in Deutschland. Wiesbaden: Statistisches Bundesamt.

Thomas, Tanja; Krotz, Friedrich (2008). «Medienkultur und Soziales Handeln: Begriffsarbeiten zur Theorieentwicklung.» Medienkultur und soziales Handeln. Hrsg. von Tanja Thomas. Wiesbaden: VS-Verlag. 17-42.

Thomas, Tanja (Hrsg.) (2008). Medienkultur und soziales Handeln. Wiesbaden: VSVerlag.

Tyrell, Hartmann (1986). «Geschlechtliche Differenzierung und Geschlechterklassifikation.» Kölner Zeitschrift für Soziologie und Sozialpsychologie. 38. Jg. H. 3. 450-489.

Wagner, Ulrike; Theunert, Helga (Hrsg.) (2006). Neue Wege durch die konvergente Medienwelt. Studie im Auftrag der Bayrischen Landeszentrale für neue Medien (BLM). München: Reinhard Fischer.

Wetterer, Angelika (2004). «Konstruktionen von Geschlecht: Reproduktionsweisen der Zweigeschlechtlichkeit.» Handbuch Frauen- und Geschlechterforschung. Theorie, Methoden, Empirie. Hrsg. von Ruth Becker u. Beate Kortendiek. Wiesbaden: VS-Verlag. 122-131.

Winter, Carsten u. a. (Hrsg.) (2003). Medienidentitäten. Identität im Kontext von Globalisierung und Medienkultur. Köln: Halem.

Wilk, Nicole M. (2002). Körpercodes. Die vielen Gesichter der Weiblichkeit in der Werbung. Frankfurt/Main: Campus. 
Willems, Herbert; Jurga, Martin (1998). «Globalisierung, medienkulturelle Tradierung und die Darstellung der Geschlechter in der Werbung.» Kommunikation im Wandel. Zur Theatralität der Medien. Hrsg. von Udo Göttlich u. a. Köln: Halem. 169-191.

Willems, Herbert; Kautt, York (1999). «Zur Codierung des Körpers: Geschlecht, Gesellschaft und Kultur.»SOCIOLOGIA INTERNATIONALIS. Internationale Zeitschrift für Soziologie. Bd. 37. H. 1/2. 131-156.

Willems, Herbert; Kautt, York (2000). «Aspekte und Konzepte einer Kultursoziologie des Körpers: Dargestellt am Beispiel der Analyse der Werbungstheatralität.» Schweizerische Zeitschrift für Soziologie. Vol. 26. Nr. 2. 345-372.

Willems, Herbert (2009). Theatralisierung der Gesellschaft. Bd. 2: Medientheatralität und Medientheatralisierung. Wiesbaden: VS-Verlag (im Erscheinen).

Zurstiege, Guido (1998). Mannsbilder - Männlichkeit in der Werbung: Zur Darstellung von Männern in der Anzeigenwerbung der 50er, 70er und 90er Jahre. Opladen, Wiesbaden: Westdeutscher Verlag. 